40 hours worked beginning in 2012. One previous study using a national survey found that workers who had paid sick leave were less likely to sustain nonfatal occupational injuries compared to those without paid sick leave. This study seeks to determine whether there has been a decrease in the rate of occupational injuries and illnesses in Connecticut following the introduction of their paid sick leave policy.

Methods Data about the overall rate of occupational illnesses and injuries and occupation and industry specific rates were obtained from the Bureau of Labour Statistics' Survey of Occupational Injuries and Illnesses for Connecticut and compared to rates for the United States overall and five other northeastern states. For all of the states and for the United States, the change in the average annual rate of occupational injuries and illnesses was compared for the four years before and after the introduction of Connecticut's paid sick leave policy.

Results The decline in the rate of occupational injuries and illnesses per 100 full-time workers was greater in Connecticut (17.1\%, 95\% Confidence Interval $=9.5 \%$ to $24.6 \%)$ than the decline in any of the other states or the United States overall. The percent decline differed by occupation and industry.

Conclusion There seems to have been an impact of Connecticut's paid sick leave policy on the rate of occupational injuries and illnesses pointing to potential additional benefits of these policies. Studying the impact of other states' paid sick leave policies will be important in the future.

\section{FURTHER DEVELOPING THE PSYCHOSOCIAL SAFETY CLIMATE TOOL BY APPLYING MIXED METHODS}

Maren Formazin*, Michael Ertel. Federal Institute for Occupational Safety and Health (BAA), Berlin, Germany

\subsection{6/oemed-2018-ICOHabstracts. 1649}

Introduction Psychosocial Safety Climate (PSC) - a specific dimension of organisational climate - refers to shared perceptions regarding policies for the protection of worker psychological health and safety (Hall, Dollard \& Coward, 2010) and is considered to comprise four content domains. The objective of the current study is to further develop the PSC instrument by investigating its validity, i.e. the comprehensibility of the PSC items, in another culture and language than where it was originally developed.

Methods The study follows a mixed-method approach. After translating the original English PSC items into German, they were revised by experts on work and health, focusing on the meaning and practical use of terms, the frame of reference and differences in legislation. To obtain a wider coverage of the underlying dimensions, further items were added.

Cognitive interview techniques (think-aloud, probing) with 25 employees of different occupations, tenure and age were applied on this enhanced version of the PSC instrument, leading to a preliminary revised PSC tool.

In the quantitative main study currently underway, this revised PSC tool is applied in a sample of more than 2000 employees from different organisations and branches to test the structure of the instrument, its assumed multi-level nature and its relations with other psychosocial working conditions and relevant health measures.
Result The cognitive interviews indicated that the term ,psychological health' is not well understood by German employees and requires a definition. Moreover, several items required adaptions in wording to ensure similar understanding among employees with different backgrounds. Preliminary results from the main study support the tool's reliability and validity in the German context.

Discussion This study demonstrates the benefits of applying both cognitive interview techniques and a quantitative survey in instrument adaptation and testing. The revised PSC tool will allow for a stronger cross-cultural use of the PSC concept.

\section{DO PERCEPTIONS OF CONTROL INFLUENCE INDUSTRIAL WORKERS' ENGAGEMENT IN OCCUPATIONAL HEALTH AND SAFETY?}

Julie MC Staun*. Independent Consultant Jægerspris Denmark

\subsection{6/oemed-2018-ICOHabstracts. 1650}

Introduction This investigation focused on perceptions of control and its potential influence on engagement in occupational health and safety by industrial workers, and highlight the much needed attention to this important group of employees. The study aimed to address the main question of the research: to what extent do perceptions of control explain the engagement in occupational health and safety by industrial workers, and what are the perceived barriers to sustainable engagement experienced by this group of workers?

Methods The mixed method quantitative-qualitative approach aimed to identify how the value and impact of the locus of control external/powerful others versus the intrinsic values perceived to be held by an individual, can contribute to the perceived causes and barriers to sustainable engagement in health and safety by workers in a contemporary industrial setting

Two variants of the locus of control construct were selected as the measurement tools for the quantitative investigation. The multidimensional Internal-Powerful Others-Chance (IPC), (Levenson 1973) scale to assess the generic personality orientations, and the domain specific Work Locus of Control scale (WLCS), (Spector 1988) was selected to identify potential context specific criteria.

Following the results of the quantitative survey qualitative in-depth interviews were conducted to detect potential influences perceived by the workers to engagement in health and safety.

Results The quantitative findings $(n=167)$ identified an incongruity between the inherent internality of the IPC scales and the internality variable of the WLCS suggesting an internal orientated workforce demonstrating less internality at the workplace. Interviews $(\mathrm{n}=10)$ provided in-depth perspectives including potential barriers to sustainable engagement in health and safety activities.

Conclusions The quantitative findings indicate perceptions of control amongst an industrial workforce could relate to the engagement in occupational health and safety, and the qualitative interviews allowed for identification of perceptions related to occupational health and safety as experienced by this group of workers 